\title{
Blood pressure changes in dogs with babesiosis
}

\author{
LS Jacobson ${ }^{\mathrm{a}}$, R G Lobetti ${ }^{\mathrm{a}}$ and T Vaughan-Scott ${ }^{\mathrm{a}}$
}

\begin{abstract}
Systemic arterial blood pressures were measured in 30 dogs with acute babesiosis, 10 each with mild uncomplicated, severe uncomplicated and complicated disease. Ten healthy dogs were used as controls. Hypotension was defined as more than 3 standard deviations below the control mean. Normal mean pressures $( \pm \mathrm{SD})$ were: systolic arterial pressure 151 $( \pm 11) \mathrm{mm} \mathrm{Hg}$, diastolic arterial pressure $89( \pm 8) \mathrm{mm} \mathrm{Hg}$ and mean arterial pressure 107 $( \pm 10) \mathrm{mm} \mathrm{Hg}$. Hypotension was the most frequent abnormality, and increased strikingly in incidence as disease severity increased, with 5/10 dogs in the complicated group being hypotensive for systolic, diastolic and mean arterial pressures, compared with $2 / 10$ in the severe uncomplicated group and 0/10 in the mild uncomplicated group. Systolic, diastolic and mean arterial pressures in the complicated group and severe uncomplicated group, and systolic pressure in the mild uncomplicated group, were significantly lower than in the controls. There were no significant relationships between arterial pressures and age, pulse rate, respiratory rate, temperature, mucous membrane colour or haematocrit. There was a significant negative correlation between arterial pressures and white cell and immature neutrophil counts. Arterial pressures differed significantly between dogs that were clinically collapsed and those that were not, but not between survivors and non-survivors. Pulse pressure (systolic - diastolic) was low in 7/10 complicated, 1/10 mild uncomplicated, and 1/10 severe uncomplicated cases, and differed significantly between the complicated and control groups. The high incidence of hypotension in clinically severe babesiosis has important implications for therapy.
\end{abstract}

Key words: Babesia canis, babesiosis, blood pressure, canine, dog, hypotension, shock.

Jacobson L S, Lobetti R G, Vaughan-Scott $T$ Blood pressure changes in dogs with babesiosis. Journal of the South African Veterinary Association (2000) 71(1): 14-20 (En.). Department of Medicine, Faculty of Veterinary Science, University of Pretoria, Private Bag X04, Onderstepoort, 0110 South Africa.

\section{INTRODUCTION}

Circulatory failure and shock have long been recognised in severe and terminal babesiosis caused by Babesia canis $^{18}$, and it has been suggested that virulent babesiosis results in 2 syndromes, one characterised by hypotensive shock and the other by haemolytic anaemia ${ }^{31}$. However, objective evidence of hypotension has been provided in only 1 case $^{10}$. In addition, little consideration has been given to the possibility that clinically mild or inapparent hypotension (as opposed to life-threatening hypotensive shock, i.e. hypotension accompanied by failure of capillary perfusion ${ }^{21}$ ) might occur in canine babesiosis, and that a fall in blood pressure might follow a continuum of severity, rather than being exclusively a fulminant marker of catastrophe.

Wright and Kerr $^{38}$ documented arterial hypotension in splenectomised calves infected with Babesia bovis, and commented that hypotensive shock was

aDepartment of Medicine, Faculty of Veterinary Science, University of Pretoria, Private Bag X04, Onderstepoort, 0110 South Africa.

Received: February 1999. Accepted: December 1999. characteristic of the disease. Blood pressure began to fall approximately 3 days after infection, well before the reduction in haematocrit $(\mathrm{Ht})$ that occurred on about day 5 , and continued to decrease over the next 6 days, until the study ended. Thus, reduced systemic blood pressure was present long before hypotensive shock occurred. Wright ${ }^{37,38}$ attributed hypotension in babesiosis to vasoactive mediators (kallikrein and kinins). However, Gilles demonstrated a marked reduction in blood volume in a dog infected with B. canis that developed shock and haemoconcentration ${ }^{11}$.

Falciparum malaria in humans is clinically similar to canine babesiosis in many respects $^{14,18,19}$. Orthostatic hypotension is common in uncomplicated malaria ${ }^{30}$. Patients with malaria typically present with increased cardiac output, low systemic vascular resistance and low to normal blood pressure ${ }^{36}$. Hypotensive shock is a relatively rare event, and is termed 'algid malaria' ${ }^{\prime 36}$.

Sepsis has been redefined in human medicine as the systemic response to infection, regardless of whether that infection is bacterial, viral, fungal or protozoal $^{6}$. The systemic response to severe insults, labelled the systemic inflammatory response syndrome (SIRS) in the absence of infection, and sepsis in its presence, is defined as 2 or more of the following: hypo- or hyperthermia, tachycardia, hyperventilation, and leukocytosis, leukopaenia or neutrophilic left shift. Acute babesiosis is thus a form of sepsis, according to the consensus definition, and would be expected (as predicted $^{7,8}$ ) to manifest in similar ways. Hypotension is a consistent feature of the haemodynamic response to sepsis in the $\mathrm{dog}$, occurring in natural ${ }^{16}$ and experimental ${ }^{29}$ bacterial sepsis, as well as after injection of endotoxin and pro-inflammatory cytokines $^{22,23}$.

The aims of this study were: (1) to measure systemic arterial pressures in dogs with naturally occurring babesiosis, (2) to establish whether the presence and severity of hypotension increases with increased disease severity; and (3) to establish whether blood pressure is correlated with easily measured clinical and/or laboratory parameters.

\section{MATERIALS AND METHODS}

Thirty dogs presented at the Onderstepoort Veterinary Academic Hospital with clinical signs of acute babesiosis were prospectively studied. Inclusion criteria were: Babesia canis parasites identified on a thin capillary blood smear, no history of an acute inflammatory or infectious disease in the past 6 weeks, and body mass greater than $5 \mathrm{~kg}$. Dogs with known (smear-positive) concurrent Ehrlichia canis infections were excluded. Informed consent of the owner was required to enrol a patient in the study. The dogs were classified into 3 groups, based on WHO criteria for severe falciparum malaria $^{35}$ and a classification system for canine babesiosis ${ }^{14}$ : (1) Mild uncomplicated babesiosis: $\mathrm{Ht} \geq 0.20 / /$, no complications; (2) severe uncomplicated babesiosis: Ht < 0.20 e/l, no complications; (3) complicated babesiosis: acute renal failure, cerebral babesiosis, haemoconcentration ('red biliary'), hepatopathy, hypoglycaemia and/or pulmonary oedema, defined as follows:

- Acute renal failure: serum creatinine 
$>150 \mu \mathrm{mol} / \mathrm{l}$ and/or oliguria $(<1 \mathrm{~m} /$ $\mathrm{kg}$ /hour); not resolved by rehydration.

- Cerebral babesiosis: central neurological signs not attributable to any other cause.

- Haemoconcentration: haematocrit $>0.37 \mathrm{l} / \mathrm{l}$ in conjunction with congested mucous membranes and severe haemolysis (severe haemoglobinuria and/or grossly visible haemoglobinaemia).

- Hepatopathy: icterus and/or alanine transaminase and alkaline phosphatase both $>2 \times$ normal upper limit.

- Hypoglycaemia: plasma glucose $\geq 2.2$ $\mathrm{mmol} / \mathrm{l}$.

- Pulmonary oedema: dyspnoea with typical frothy nasal discharge, radiographic oedema and/or $\mathrm{P}_{\mathrm{a}} \mathrm{O}_{2}<60$ $\mathrm{mm} \mathrm{Hg}$.

The control group consisted of unmatched healthy dogs from the same catchment area as the dogs with babesiosis, admitted for routine sterilisation. To qualify for inclusion, these dogs had to be clinically normal and aparasitaemic. There were $10 \mathrm{dogs}$ in each group.

A full clinical examination was performed at admission. Blood samples and blood pressure measurements were obtained before treatment was administered. Blood pressure was determined non-invasively by an oscillometric technique, using a Dinamap blood pressure monitor (Critikon, Johnson \& Johnson) and neonatal cuffs (Disposa-Cuf, Critikon). Each dog was positioned in right lateral recumbency and the cuff placed on the left distal forelimb immediately proximal to the carpus, over the radial artery. The cuff size was selected so that the width was approximately $40 \%$ of the limb circumference. Five to 6 readings of systolic arterial pressure (SAP), diastolic arterial pressure (DAP) and mean arterial pressure (MAP) were recorded over a 5-minute period. The readings were then averaged. The Dinamap measures SAP and DAP, and calculates MAP. Pulse pressure (PP) was calculated by subtracting DAP from SAP. The laboratory database consisted of haematology and serum biochemistry (urea, creatinine, bilirubin, alanine transaminase, alkaline phosphatase and glucose).

All dogs with babesiosis were treated with diminazene aceturate (Berenil, Hoechst) according to the manufacturer's instructions. Dogs with mild uncomplicated babesiosis did not receive additional supportive treatment. Dogs with $\mathrm{Ht}<0.15 \mathrm{l} / \mathrm{c}$ were given a whole blood transfusion. Other supportive treatment was tailored to individual requirements and was not standardised.

The results were analysed using Excel
$97^{\mathrm{TM}}$ (Microsoft) and SigmaStat ${ }^{\mathrm{TM}}$ v2.0.3 (Jandel Scientific). Hyper- and hypotension were defined as falling outside 3 standard deviations (SD) of the normal control group $^{32}$. Values greater or less than 2 SD and up to 3 SD from the mean were considered borderline ${ }^{32}$. Nonparametric statistical methods were used for most comparisons, for 2 reasons: (1) the small sample size meant that normal distribution of data could not be established with certainty; and (2) although most blood pressure data in this study was normally distributed, a large study has shown that blood pressures in dogs have a lognormal distribution ${ }^{5}$. The Mann-Whitney rank sum test was used for comparisons between 2 groups, and Kruskal-Wallis ANOVA on ranks, with pairwise comparisons against the control group using Dunnett's method, for comparisons between 3 or more groups. The StudentNeuman-Keuls test was used for pairwise comparisons among the babesiosis groups. The Spearman rank order correlation was used to determine the strength of association between selected variables in the patient groups. Where variables from all 30 patients were used for correlation or regression analysis, data were also analysed using the Pearson product moment correlation or linear regression, after normalisation where required. Univariate analysis was used to measure relationships between grouped variables in the patient groups, using the MannWhitney and Kruskal-Wallis tests. The probability value for significance was set at $P<0.05$.

\section{RESULTS}

Signalment, clinical and laboratory data for the study population are summarised in Table 1 and blood pressure data shown in Table 2. The signalment of the control group was similar to that of the babesiosis groups. Laboratory values were frequently abnormal in the babesiosis groups, and often differed from controls and between groups (see Table 2). The abnormalities were consistent with the disease classification. In the complicated group, 3 dogs had acute renal failure (Dogs 3, 7 and 9); 4 had haemoconcentration (Dogs 3, 5, 6 and 7); 2 had icterus (Dogs 4 and 8); 3 had hypoglycaemia (Dogs 1, 2 and 10) and 2 had pulmonary oedema (Dogs 3 and 5). Dog 9 also had rhabdomyolysis. No dog had cerebral babesiosis. The dogs with visible icterus had extremely high bilirubin concentrations (142.6 and $165.6 \mu \mathrm{mol} / l$, respectively), but no dogs had both liver enzymes elevated above twice normal. Hypoglycaemia has not previously been recognised in canine babesiosis, and will be reported in more detail separately, together with other data on carbohydrate metabolism.

Normal ranges for blood pressures, obtained from the control group, were as follows (mean ( \pm 2 SD)): SAP 151 (129-173) mm Hg; DAP 89 (73-105) mm Hg; MAP 107 (86-128) mm Hg; and PP 62 (48-76) $\mathrm{mm} \mathrm{Hg}$. The cut-off values for hypertension were SAP $>184 \mathrm{~mm} \mathrm{Hg}$; DAP > $113 \mathrm{~mm} \mathrm{Hg}$; and MAP > 138 $\mathrm{mm} \mathrm{Hg}$, and for hypotension SAP $<118 \mathrm{~mm} \mathrm{Hg}$; DAP <65 mm Hg; and MAP $<76 \mathrm{~mm} \mathrm{Hg}$. Cut-off values for elevated and low PP were, respectively, $>83 \mathrm{~mm} \mathrm{Hg}$ and $<41 \mathrm{~mm} \mathrm{Hg}$.

Hypotension was the most common abnormality, and increased strikingly in incidence as disease severity increased (Table 2A-D). Five of 10 dogs in the complicated group had hypotensive values for SAP, DAP and MAP, compared with $2 / 10$ in the severe uncomplicated group and $0 / 10$ in the mild uncomplicated group. Eight of the 10 dogs in the complicated group had at least 1 hypotensive value, compared with $6 / 10$ in the severe uncomplicated group and $3 / 10$ in the mild uncomplicated group. As a rule, changes in a particular pressure measurement were paralled by changes in the others, although the degree of change frequently varied. Pulse pressures were abnormally low in $7 / 10$ dogs in the complicated group, $1 / 10$ in the mild uncomplicated group and 1/10 in the severe complicated group. The changes in PP indicated that SAP and DAP did not tend to change by the same amount. Elevated arterial pressures were uncommon, with only $1 \mathrm{dog}$ (mild uncomplicated group Dog 8) showing hypertension (elevated SAP, DAP and MAP). DAP and MAP were elevated in Dog 5 in the complicated group, and borderline elevated SAP was present in Dog 5 in the mild uncomplicated group.

There was a significant difference in SAP $(P<0.05)$ between all 3 patient groups and the control group (Fig. 1A). The DAP and MAP of the severe uncomplicated and complicated groups, but not of the mild uncomplicated group, differed significantly from the controls $(P<$ 0.05 ) (Fig. 1B-C). There was a significant difference in PP between complicated cases and controls (Fig. 1D).

There were no significant relationships between SAP, DAP or MAP and age, pulse rate, respiratory rate, rectal temperature and Ht. There was no correlation between $\mathrm{PP}$ and Ht. A significant negative correlation was found between white cell count and SAP, DAP and MAP (SAP: $r^{2}=0.252$, $P=0.0049$; DAP: $r^{2}=0.297, P=0.00195$; MAP: $\left.r^{2}=0.2959, P=0.0020\right)$. Results were similar using normalised data and 


\begin{tabular}{|c|c|c|c|c|c|}
\hline & $\begin{array}{l}\text { Normal values }^{\dagger} \\
\text { (where applicable) }\end{array}$ & Mild uncomplicated & Severe uncomplicated & Complicated & Control \\
\hline Age (years) & & $2.9(3.0)$ & $3.5(4.2)$ & $2.5(2.7)$ & $2.1(1.3)$ \\
\hline $\operatorname{Sex}(M: F)$ & & $6: 4$ & $3: 7$ & $6: 4$ & $3: 7$ \\
\hline Weight (kg) & & $24.6(14.5)$ & $17.3(8.7)$ & $24.0(15.6)$ & $18.2(8.3)$ \\
\hline Rectal temperature $\left({ }^{\circ} \mathrm{C}\right)$ & $38.4-39.4^{1}$ & $40.0(0.8)$ & $39.8(0.6)$ & $39.4(1.3)$ & NR \\
\hline Pulse rate (per min) & $70-120^{1}$ & $118(27)$ & $132(23)$ & $119(42)$ & NR \\
\hline Respiratory rate (per min) & $18-34^{1}$ & $53(24)$ & $49(21)$ & $41(10)$ & NR \\
\hline $\begin{array}{l}\text { Mucous membrane colour } \\
\text { (normal:pale:congested:icteric) }\end{array}$ & & $3: 6: 1: 0$ & $0: 10: 0: 0$ & $0: 3: 5: 2$ & 10:0:0:0 \\
\hline Haematocrit $(\ell / \ell)$ & $0.37-0.55$ & $0.34(0.08)^{\star a}$ & $0.13(0.03)^{* a b}$ & $0.27(0.20)^{\star b}$ & $0.50(0.04)$ \\
\hline White cell count $\left(\times 10^{9} / \ell\right)$ & $6.0-15.0$ & $5.5(1.9)^{\star a}$ & $8.6(4.3)^{\star a}$ & $13.2(7.3)^{\mathrm{a}}$ & $13.3(6.7)$ \\
\hline Immature neutrophil count $\left(\times 10^{9} / \ell\right)$ & $0-0.30$ & $0.29(0.54)^{\mathrm{ab}}$ & $1.27(1.53)^{* a}$ & $1.88(2.84)^{* b}$ & $0.28(0.41)$ \\
\hline Urea $(\mathrm{mmol} / \ell)$ & $3.6-8.9$ & $6.8(3.5)^{\mathrm{ab}}$ & $13.3(6.5)^{\star a}$ & $18.7(6.4)^{\star b}$ & $6.7(2.6)$ \\
\hline Creatinine $(\mu \mathrm{mol} / \ell$ & $<133$ & $104(30)^{\star} \mathrm{ab}$ & $84(25)^{\star}$ ac & $146(61)^{b c}$ & $123(16)$ \\
\hline Total bilirubin $(\mu \mathrm{mol} / \ell)$ & $<6.8$ & $10.0(11.8)$ & $20.5(23.8)$ & $49.3(59.6)$ & $14.4(13.8)$ \\
\hline Alanine transaminase $(\mathrm{U} / \ell)$ & $<40$ & $21(6)$ & $48(74)$ & $50(43)$ & $23(6)$ \\
\hline Alkaline phosphatase $(\mathrm{U} / \ell)$ & $<190$ & $77(40)^{*}$ & $178(295)^{*}$ & $84(59)^{*}$ & $44(23)$ \\
\hline Glucose $(\mathrm{mmol} / \mathrm{l})$ & $3.3-5.5$ & $5.1(0.6)^{\mathrm{a}}$ & $5.1(0.8)^{b}$ & $3.5(2.0)^{\star} \mathrm{ab}$ & $5.2(0.7)$ \\
\hline Collapsed at presentation & & 0 & 2 & 7 & 0 \\
\hline Died & & 0 & 1 & 4 & 0 \\
\hline
\end{tabular}

${ }^{\dagger}$ Normal values are those used at the Onderstepoort Veterinary Academic Hospital, except where indicated otherwise.

*Significantly different from control group. Patient groups with the same superscripts $(\mathrm{a}, \mathrm{b}$ or $\mathrm{c})$ differ significantly from one another $(P<0.05)$.

parametric methods. A significant negative correlation was found between SAP, DAP and MAP and immature neutrophil count using non-parametric statistics (SAP: $r^{2}=0.1354, P=0.0455$; DAP: $r^{2}=$ 0.1918, $P=0.0157$; MAP: $r^{2}=0.1998 ; P=$ $0.0135)$, but the significance was lost when parametric methods and normalised data were used, although in all cases $P$ still tended towards significance.

Blood pressure variables were compared between groupings based on clinical variables, to establish whether any of these might be predictive of hypotension. There were no significant differences in blood pressures between patients grouped according to respiratory abnormalities ( $\leq 40$ breaths per minute and $>40$ breaths per minute/panting/ dyspnoea); $\mathrm{Ht}(<0.20 \mathrm{l} / \mathrm{l}$ and $>0.20 \mathrm{l} / \mathrm{l})$; rectal temperature $\left(<37.5^{\circ} \mathrm{C} ; 38.5-40{ }^{\circ} \mathrm{C}\right.$; and $\left.>40{ }^{\circ} \mathrm{C}\right)$; or age group $(<0.5$ years; $0.5-5$ years; and $>$ 5years). Blood pressure did not differ according to mucous membrane colour, whether this was grouped as normal vs pale $v s$ icteric $v s$ congested; or pale vs non-pale; or congested vs non-congested. There was a trend towards a significant difference between pale and non-pale mucous membranes for SAP (median (25th-75th percentile): Pale $135 \mathrm{~mm} \mathrm{Hg}$ (112-148); non-pale $107 \mathrm{~mm} \mathrm{Hg}$ ( 87-122); $P=0.055)$. There was no relationship between PP and $\mathrm{Ht}$ when dogs were divided into severely anaemic ( $\mathrm{Ht}<0.20 \mathrm{l} / \mathrm{l}$ ), moderately or mildly anaemic (Ht $0.20-0.37 \ell / \ell$ ) and non-anaemic (Ht $>0.37$ l/l) groups.

Although there were no significant differences in blood pressures between dogs that survived $(n=25)$ and non-survivors $(n=5)$, a trend towards significance was present for DAP (median (25th-75th percentile): survivors $73 \mathrm{~mm} \mathrm{Hg}$ (60-89); non-survivors $58 \mathrm{~mm} \mathrm{Hg}$ (49-67); $P=$ 0.080 ). Five dogs died (Dog 2 in the severe uncomplicated group and Dogs 1, 2, 4 and 6 in the complicated group). Four had severe anaemia and 1 had haemoconcentration. Two of these dogs (severe uncomplicated group Dog 2 and complicated group Dog 2) had abnormally low SAP, DAP and MAP, while the other 3 each had at least 1 hypotensive value. DAP was low in $4 / 5$ non-survivors.

There was a significant difference between patients described as collapsed on clinical examination $(n=9)$ and those not considered to be collapsed $(n=21)$ for SAP, DAP and MAP $(P<0.001)$, as well as for PP $(P<0.05)$ (Fig. 2). Most, but not all, collapsed dogs had hypotension.

\section{DISCUSSION}

The results of this study support the usefulness of indirect blood pressure measurement as a clinical tool. The cut-off points used for abnormal values ${ }^{32}$ identified substantially more dogs as hypotensive than a more conservative text- book definition ${ }^{17}$. Using the textbook definition of hypotension (SAP $<80 \mathrm{~mm} \mathrm{Hg}$ or MAP $<60 \mathrm{~mm} \mathrm{Hg}^{17}$ ), the number of dogs with hypotension dropped substantially, with only 3 , all in the complicated group (Dogs 2,7 and 8), fitting the criteria. Since hypotension is only clinically significant when it is associated with poor tissue perfusion ${ }^{17}$, the conservative definition might be more correct; however, tissue perfusion cannot be measured in the routine clinical environment and if hypotension is identified early and treated, perfusion failure and shock might be avoided. There is thus much to commend the less conservative definition. It is advisable for each institution to establish its own normal values, since values differ between studies, even those using the same equipment $t^{5,32,34}$.

The changes in blood pressure in the severely ill dogs with babesiosis were not surprising, given the clinical evidence of collapse and shock in many of these animals. It is notable, however, that even in the mild uncomplicated group, SAP was significantly lower than that of the control group. The low blood pressures in the severely anaemic group were somewhat unexpected, since severe anaemia should result in increased cardiac output and stroke volume in order to maintain tissue oxygenation. It therefore appears that, at least in some dogs, these compensatory mechanisms falter, possibly as a 
Table 2: Systemic arterial pressures in dogs with babesiosis.

\begin{tabular}{|c|c|c|c|c|c|c|c|c|}
\hline Dog & \multicolumn{2}{|c|}{ Mild uncomplicated } & \multicolumn{2}{|c|}{ Severe uncomplicated } & \multicolumn{2}{|c|}{ Complicated } & \multicolumn{2}{|c|}{ Control } \\
\hline \multicolumn{9}{|c|}{ A: systolic arterial pressure $(\mathrm{mm} \mathrm{Hg})$} \\
\hline 1 & 147 & 0 & 133 & $O$ & 135 & $\boldsymbol{\nabla}$ & 140 & 0 \\
\hline 2 & 123 & $\square$ & 105 & $\nabla$ & 84 & $\boldsymbol{\nabla}$ & 150 & $\mathrm{O}$ \\
\hline 3 & 147 & $\mathrm{O}$ & 135 & $\mathrm{O}$ & 87 & $\nabla$ & 160 & $\bigcirc$ \\
\hline 4 & 101 & $\nabla$ & 110 & $\boldsymbol{\nabla}$ & 119 & $\square$ & 160 & $\bigcirc$ \\
\hline 5 & 176 & $\mathbf{\square}$ & 80 & $\nabla$ & 156 & $\bigcirc$ & 155 & $\bigcirc$ \\
\hline 6 & 143 & $\bigcirc$ & 116 & $\boldsymbol{\nabla}$ & 107 & $\nabla$ & 170 & $\bigcirc$ \\
\hline 7 & 102 & $\nabla$ & 158 & O & 80 & $\nabla$ & 155 & O \\
\hline 8 & 191 & $\boldsymbol{\Delta}$ & 148 & O & 82 & $\nabla$ & 140 & O \\
\hline 9 & 111 & $\nabla$ & 118 & $\square$ & 88 & $\nabla$ & 138 & 0 \\
\hline 10 & 152 & $O$ & 148 & 0 & 123 & O & 139 & 0 \\
\hline Median & \multicolumn{2}{|c|}{$145^{\star}$} & \multicolumn{2}{|c|}{$126^{*}$} & \multicolumn{2}{|c|}{$98^{*}$} & \multicolumn{2}{|c|}{153} \\
\hline 25th percentile & \multicolumn{2}{|c|}{111} & \multicolumn{2}{|c|}{110} & \multicolumn{2}{|c|}{84} & \multicolumn{2}{|c|}{140} \\
\hline 75th percentile & \multicolumn{2}{|c|}{152} & \multicolumn{2}{|c|}{148} & \multicolumn{2}{|c|}{123} & \multicolumn{2}{|c|}{160} \\
\hline
\end{tabular}

\section{B: diastolic arterial pressure $(\mathrm{mm} \mathrm{Hg})$}

\begin{tabular}{|c|c|c|c|c|c|c|c|c|}
\hline 1 & 92 & O & 78 & $\mathrm{O}$ & 64 & $\nabla$ & 85 & O \\
\hline 2 & 66 & $\square$ & 50 & $\nabla$ & 47 & $\nabla$ & 90 & O \\
\hline 3 & 93 & O & 64 & $\boldsymbol{\nabla}$ & 50 & $\boldsymbol{\nabla}$ & 95 & $\mathrm{O}$ \\
\hline 4 & 54 & $\nabla$ & 69 & $\square$ & 58 & $\nabla$ & 85 & $\mathrm{O}$ \\
\hline 5 & 87 & O & 58 & $\boldsymbol{\nabla}$ & 134 & $\Delta$ & 90 & $\mathrm{O}$ \\
\hline 6 & 76 & 0 & 73 & 0 & 74 & 0 & 100 & O \\
\hline 7 & 77 & O & 66 & $\square$ & 45 & $\boldsymbol{\nabla}$ & 100 & $\mathrm{O}$ \\
\hline 8 & 134 & $\Delta$ & 98 & O & 60 & $\boldsymbol{\nabla}$ & 75 & $\mathrm{O}$ \\
\hline 9 & 69 & $\square$ & 51 & $\nabla$ & 46 & $\boldsymbol{\nabla}$ & 84 & O \\
\hline 10 & 87 & ○ & 90 & ○ & 88 & ○ & 83 & $\mathrm{O}$ \\
\hline Median & \multicolumn{2}{|c|}{82} & \multicolumn{2}{|c|}{$68^{*}$} & \multicolumn{2}{|c|}{$59^{*}$} & \multicolumn{2}{|c|}{88} \\
\hline 25th percentile & \multicolumn{2}{|c|}{69} & \multicolumn{2}{|c|}{58} & \multicolumn{2}{|c|}{47} & \multicolumn{2}{|c|}{84} \\
\hline 75th percentile & \multicolumn{2}{|c|}{92} & \multicolumn{2}{|c|}{78} & \multicolumn{2}{|c|}{74} & \multicolumn{2}{|c|}{95} \\
\hline \multicolumn{9}{|c|}{ C: mean arterial pressure $(\mathrm{mm} \mathrm{Hg})$} \\
\hline 1. & 118 & O & 100 & $\square$ & 84 & $\square$ & 90 & $\mathrm{O}$ \\
\hline 2 & 89 & $\mathrm{O}$ & 75 & $\nabla$ & 57 & $\nabla$ & 100 & O \\
\hline 3 & 118 & O & 89 & O & 64 & $\nabla$ & 110 & $\mathrm{O}$ \\
\hline 4 & 80 & $\square$ & 87 & O & 85 & $\square$ & 105 & $\mathrm{O}$ \\
\hline 5 & 117 & O & 68 & $\nabla$ & 149 & $\Delta$ & 105 & $\mathrm{O}$ \\
\hline 6 & 99 & O & 92 & O & 89 & O & 120 & $\mathrm{O}$ \\
\hline 7 & 84 & $\square$ & 89 & O & 58 & $\nabla$ & 127 & $\mathrm{O}$ \\
\hline 8 & 163 & $\Delta$ & 102 & O & 54 & $\nabla$ & 100 & $\mathrm{O}$ \\
\hline 9 & 86 & $\mathrm{O}$ & 69 & $\nabla$ & 61 & $\nabla$ & 109 & $\mathrm{O}$ \\
\hline 10 & 117 & O & 110 & 0 & 111 & O & 106 & O \\
\hline Median & \multicolumn{2}{|c|}{108} & \multicolumn{2}{|c|}{$89^{*}$} & \multicolumn{2}{|c|}{$74^{*}$} & \multicolumn{2}{|c|}{106} \\
\hline 25th percentile & \multicolumn{2}{|c|}{86} & \multicolumn{2}{|c|}{75} & \multicolumn{2}{|c|}{58} & \multicolumn{2}{|c|}{100} \\
\hline 75th percentile & \multicolumn{2}{|c|}{118} & \multicolumn{2}{|c|}{100} & \multicolumn{2}{|c|}{89} & \multicolumn{2}{|c|}{110} \\
\hline
\end{tabular}

\section{D: pulse pressure $(\mathrm{mm} \mathrm{Hg})$}

\begin{tabular}{|c|c|c|c|c|c|c|c|c|}
\hline 1 & 55 & $\bigcirc$ & 55 & $\bigcirc$ & 71 & ○ & 55 & $\bigcirc$ \\
\hline 2 & 57 & $\mathrm{O}$ & 55 & $\mathrm{O}$ & 37 & $\nabla$ & 60 & $\mathrm{O}$ \\
\hline 3 & 54 & O & 71 & $\bigcirc$ & 37 & $\nabla$ & 65 & ○ \\
\hline 4 & 47 & $\square$ & 41 & $\square$ & 61 & ○ & 75 & O \\
\hline 5 & 89 & $\Delta$ & 22 & $\boldsymbol{\nabla}$ & 22 & $\nabla$ & 65 & ○ \\
\hline 6 & 67 & O & 43 & $\square$ & 33 & $\nabla$ & 70 & O \\
\hline 7 & 25 & $\nabla$ & 92 & $\boldsymbol{\Delta}$ & 35 & $\nabla$ & 55 & O \\
\hline 8 & 57 & O & 50 & O & 22 & $\nabla$ & 65 & O \\
\hline 9 & 42 & $\square$ & 67 & $\bigcirc$ & 42 & $\square$ & 54 & O \\
\hline 10 & 65 & O & 58 & $\bigcirc$ & 35 & $\nabla$ & 56 & ○ \\
\hline Median & \multicolumn{2}{|c|}{56} & \multicolumn{2}{|c|}{55} & \multicolumn{2}{|c|}{$36^{*}$} & \multicolumn{2}{|c|}{63} \\
\hline 25th percentile & \multicolumn{2}{|c|}{47} & \multicolumn{2}{|c|}{43} & \multicolumn{2}{|c|}{33} & \multicolumn{2}{|c|}{55} \\
\hline 75th percentile & \multicolumn{2}{|c|}{65} & \multicolumn{2}{|c|}{67} & \multicolumn{2}{|c|}{42} & \multicolumn{2}{|c|}{65} \\
\hline
\end{tabular}

$\mathrm{O}=$ normotensive; $\boldsymbol{\square}=$ borderline hypotensive; $\boldsymbol{\nabla}=$ hypotensive; $\boldsymbol{\nabla}=$ borderline hypertensive; $\boldsymbol{\Delta}=$ hypertensive. Borderline was defined as greater or less than 2 and up to 3 SD from control group mean, and hyper-and hypotensive as greater or less than 3 SD from the mean. Blood pressure values for control group: systolic arterial pressure $151( \pm 11) \mathrm{mm} \mathrm{Hg}$; diastolic arterial pressure $89( \pm 8) \mathrm{mm} \mathrm{Hg}$; mean arterial pressure $107( \pm 10) \mathrm{mm} \mathrm{Hg}$; pulse pressure $62( \pm 7) \mathrm{mm} \mathrm{Hg}$.

${ }^{*}$ Significantly different from control group $(P<0.05)$. 
A. Systolic arterial pressure

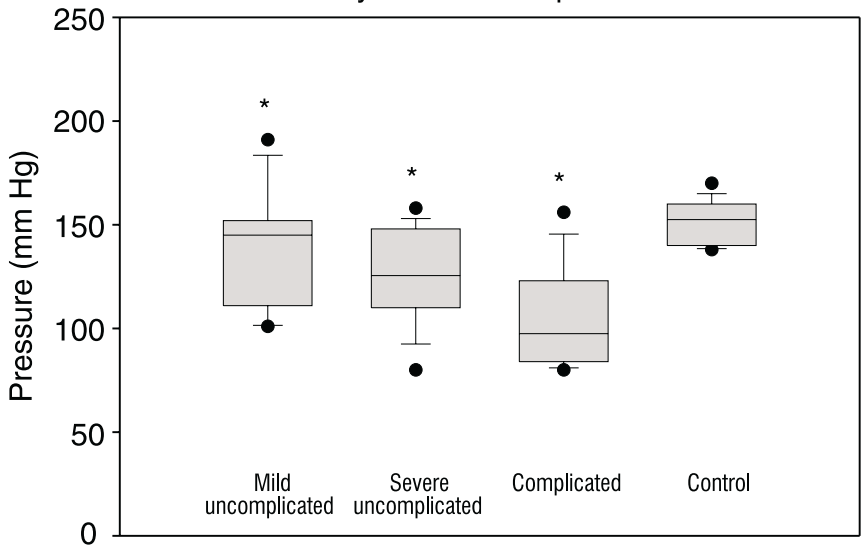

C. Mean arterial pressure

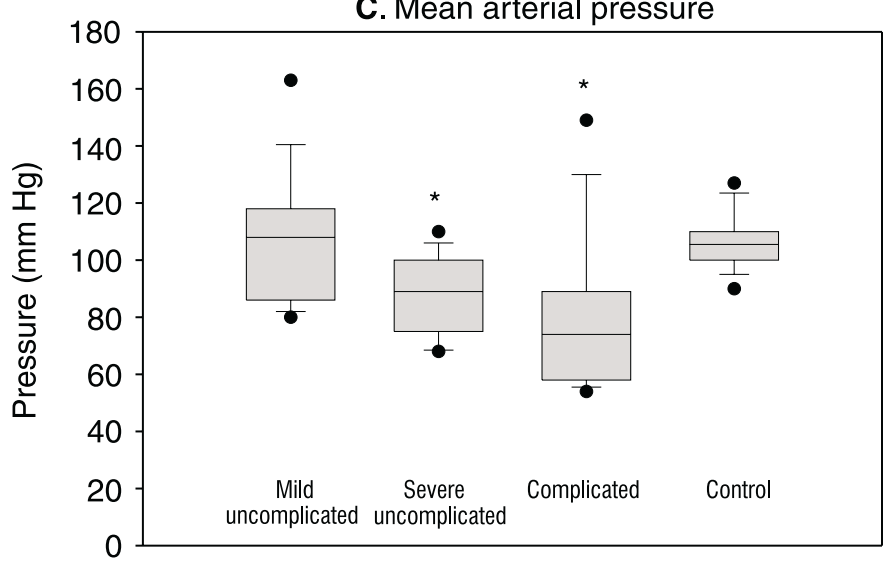

B. Diastolic arterial pressure

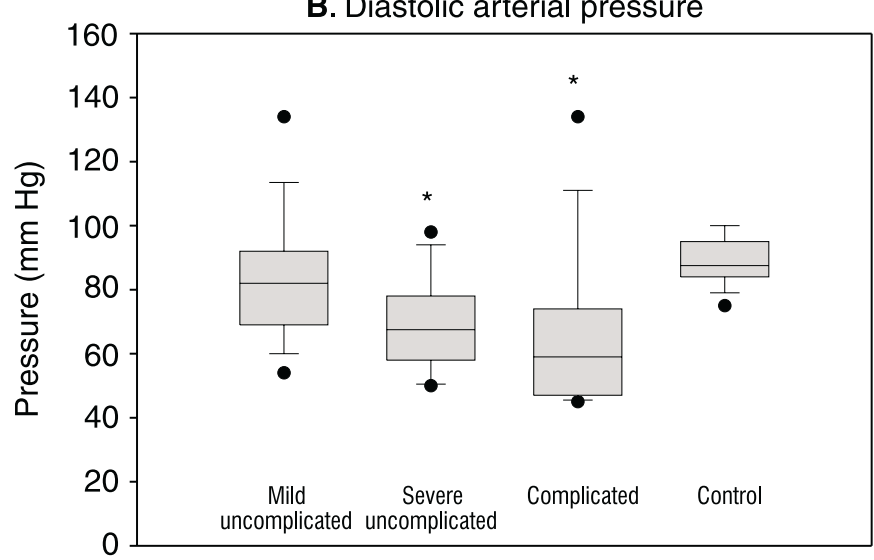

D. Pulse pressure (systolic minus diastolic)

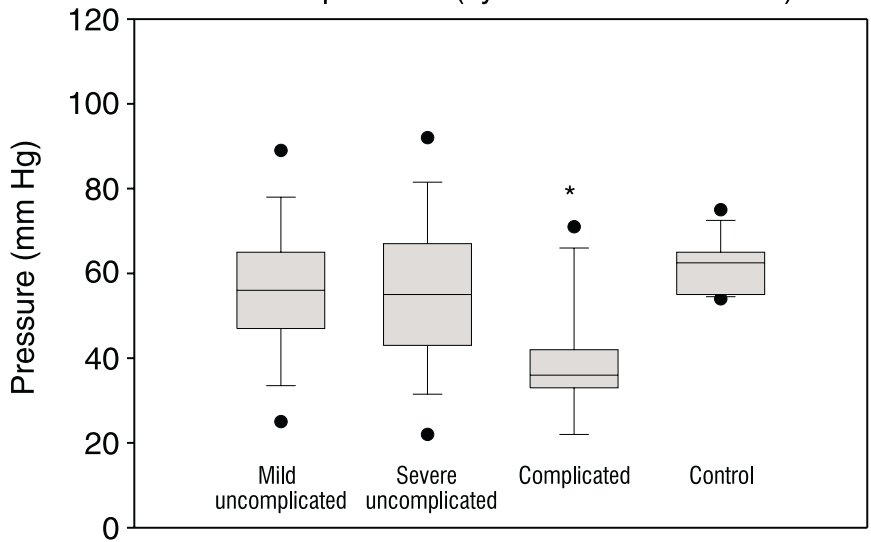

Fig 1: Systemic arterial pressures in dogs with babesiosis and healthy controls. Data are shown as median (horizontal line within box), 25th and 75th percentiles (horizontal ends of boxes), 10th and 90th percentiles (T-bars) and outliers (solid circles). ${ }^{*}=$ significantly different from control group $(P<0.05)$.

combined result of the acuteness of onset and severity of anaemia, and the inflammatory mechanisms associated with babesiosis. Viewing haemolytic anaemia and hypotensive shock as separate syndromes in babesiosis ${ }^{31}$ does not seem appropriate, given the overlap between the 2 syndromes. The presence of hypotension in a large proportion of dogs with complicated babesiosis is consistent with the hypothesis that inflammatory mechanisms play a major role in this syndrome, resulting in a sepsis-like state $\mathrm{e}^{7,14,18,25}$.

What is the cause of hypotension in babesiosis? From a cardiovascular viewpoint, the most likely mechanisms are increased capillary permeability with movement of fluid to the interstitium and/or reduced vascular tone with venous pooling. Both occur in bacterial sepsis, and can be exacerbated by myocardial depression ${ }^{12}$. Increased systemic capillary permeability occurs in malaria ${ }^{2,3}$, and its severity is associated with disease severity ${ }^{9}$. Capillary leakage in malaria is not necessarily associated with hypovolaemia, since macromolecules are returned to plasma via lymph; in fact, increased plasma volume has been reported $^{3,28}$. This is consistent with recent evidence of increased plasma volume in canine babesiosis ${ }^{26}$, possibly due to movement of interstitial fluid into the vasculature secondary to hypotension ${ }^{27}$, and with evidence of increased blood volume in the presence of reduced MAP in some patients with malaria ${ }^{28}$. The pathogenesis of this phenomenon is thought to be reduction of effective blood volume

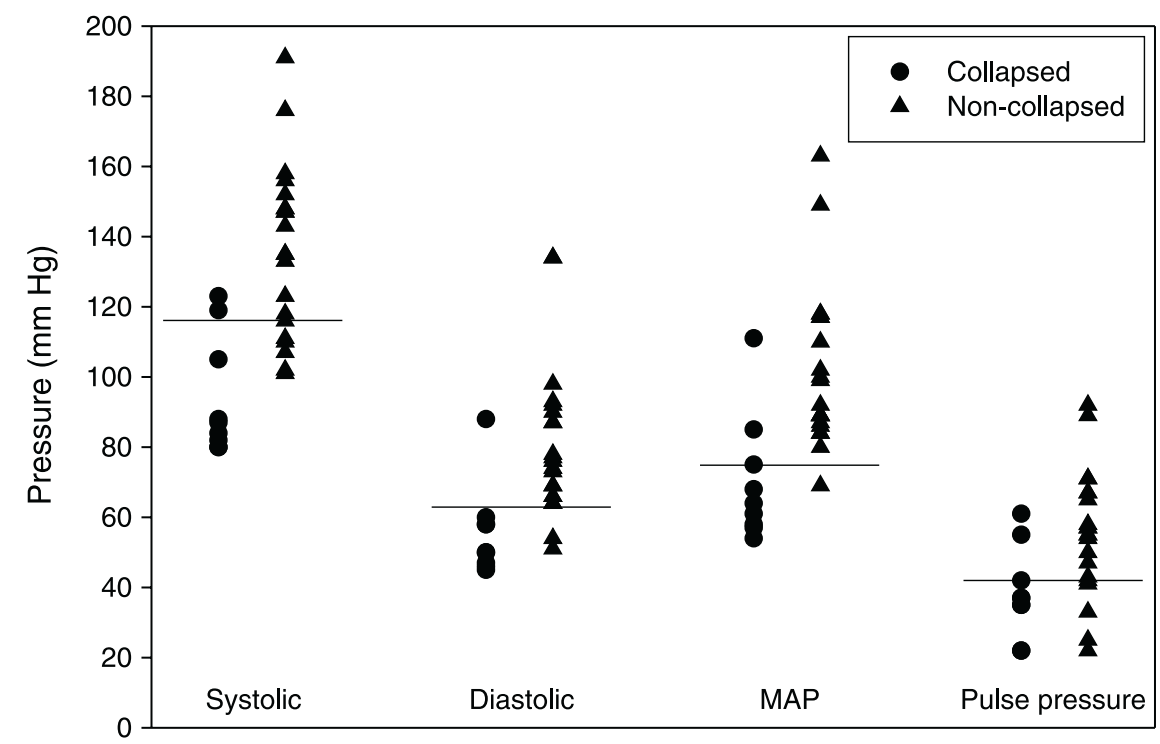

Fig 2: Systemic arterial pressures in collapsed versus non-collapsed dogs with babesiosis. Horizontal lines show lower cut-off values ( 3 standard deviations below the mean) for each

pressure. $\mathrm{MAP}=$ mean arterial pressure.

through peripheral vasodilation, followed by release of vasopressors, noradrenaline, renin activation and reduced renal haemodynamics ${ }^{28}$. Based on the above, it is likely that reduced vascular tone is the predominant mechanism of hypotension in babesiosis. As in other septic states, nitric oxide, a 'downstream' 
effector of many inflammatory mediators, and a potent and ubiquitous vasodilator $^{23}$, might play an important role.

The lack of correlation between blood pressures and most of the clinical parameters tested was unexpected. In a large study, Bodey and Michell ${ }^{5}$ showed that age accounted for almost $60 \%$ of variation in blood pressure in normal dogs, with dogs under 6 months having the lowest pressures, but age did not correlate with blood pressure in our study. Heart rate was expected to increase as blood pressure dropped, but this did not occur, nor was it a consistent finding in hypotensive dogs with endotoxaemia ${ }^{29}$ or hypotensive calves with babesiosis ${ }^{38}$. Surprisingly, PP and $\mathrm{Ht}$ were not related to one another. It was expected that the 'bounding' or 'waterhammer' pulse frequently encountered in severely anaemic dogs with babesiosis ${ }^{14}$ would be caused by a widening in PP, but the results of this study did not support this. Pulse pressure was narrowed in hypotensive dogs, reflecting, overall, a greater drop in SAP than DAP. A relationship between mucous membrane colour and blood pressure was also expected, but was not found. The slightly higher SAP in dogs with pale mucous membranes than in those without mucosal pallor could be attributed to compensatory mechanisms associated with severe anaemia. This was clearly not a uniform phenomenon, given the lack of correlation between $\mathrm{Ht}$ and blood pressure overall. The negative correlation between blood pressure and white cell count/immature neutrophil count might reflect a relationship between systemic inflammation and hypotension. An elevated white cell count and neutrophilic left shift were the most sensitive indicators of sepsis in a recent canine study ${ }^{13}$.

Blood pressures were not significantly different between dogs that survived and those that died. This is consistent with findings in dogs with septic peritonitis, in which MAP following surgery was not of prognostic value ${ }^{16}$. As in this study, pressures were lower in dogs that died than in those that survived, but the differences were not statistically significant. It is possible that blood pressure is not a predictor of mortality in babesiosis, but larger numbers would be needed to establish this. It is also possible, however, that changes in pressures over time, as opposed to a single measure, would be more useful. In humans with septic shock, MAP at admission did not differ between survivors and non-survivors, but MAP at 24 hours and the change in MAP over 24 hours were useful prognostic indicators ${ }^{4}$. The presence or absence of clinical collapse was the only clinical factor for which blood pressure measurements differed significantly. However, evaluation of collapse is subjective, and its presence or absence was not $100 \%$ sensitive or specific for hypotension (see Fig. 2).

The endpoint of hypotension is hypotensive shock. Shock is essentially a failure of capillary perfusion ${ }^{21}$. Treatment of shock in the dog has been reviewed ${ }^{12,20}$, and options for fluid therapy in canine babesiosis patients with shock have been suggested $^{15}$. In severely anaemic dogs, packed red blood cells or whole blood are the fluids of choice, while in non-anaemic hypotensive dogs, colloids are preferred to crystalloids, as crystalloids move rapidly into the interstitial space, reducing their beneficial effect on blood pressure and perfusion, and increasing the likelihood of interstitial oedema ${ }^{20}$. Central venous pressure is a good indicator of overhydration and should be monitored if possible ${ }^{20}$. Serial arterial pressure measurements are useful indicators of the efficacy of fluid therapy in the absence of more invasive techniques. Other indicators of the effectiveness of fluid therapy and resuscitation are normalisation of urine output, serum lactate concentration and base deficit ${ }^{20,24}$.

\section{CONCLUSIONS}

Hypotension, with the potential sequelae of collapse and/or hypotensive shock, should be suspected in dogs with severe uncomplicated and complicated babesiosis. The presence of clinical collapse is a good indicator of hypotension, but does not replace arterial blood pressure measurement. Prevention of shock by early monitoring of blood pressure and appropriate fluid therapy is a rational strategy in dogs with severe uncomplicated and complicated babesiosis.

\section{ACKNOWLEDGEMENTS}

Funding for this study was provided by the Pet Memorial Fund of the South African Veterinary Foundation and the Faculty of Veterinary Science of the University of Pretoria. The authors gratefully acknowledge their support. We also thank Mrs. E Viljoen, Centre for Epidemiological Research in Southern Africa, Medical Research Council, for assistance with the statistical analysis, and Dr D B Miller, Department of Medicine, Faculty of Veterinary Science, University of Pretoria, for information on blood pressure measurement.

\section{REFERENCES}

1. Aiello S E, Mays A 1998 The Merck veterinary manual (8th edn). Merck \& Company, New Jersey
2. Areekul S 1988 Transcapillary escape rate and capillary permeability to albumin in patients with Plasmodium falciparum. Annals of Tropical Medicine and Parasitology 82: 135-140

3. Areekul S, Kasemsuth R, Kanakakorn K 1984 Studies on the transcapillary escape rate of fibrinogen and capillary permeability in patients with Plasmodium falciparum malaria. Tropical and Geographical Medicine 36: 151-157

4. Bernardin G, Pradier C, Tiger F, Deloffre P, Mattei M 1996 Blood pressure and arterial lactate level are early indicators of shortterm survival in human septic shock. Intensive Care Medicine 22: 17-25

5. Bodey A R, Michell A R 1996 Epidemiological study of blood pressure in domestic dogs. Journal of Small Animal Practice 37: 116-125

6. Bone R C, Balk R A, Cerra F B, Dellinger R P, Fein A M, Knaus W A, Schein R M, Sibbald W J 1992 Definitions for sepsis and organ failure and guidelines for the use of innovative therapies in sepsis. The ACCP/SCCM Consensus Conference Committee. American College of Chest Physicians/Society of Critical Care Medicine. Chest 101: 1644 1655

7. Clark I A 1978 Does endotoxin cause both the disease and parasite death in acute malaria and babesiosis? Lancet 2: 75-77

8. Clark I A 1982 Correlation between susceptibility to malaria and babesia parasites and to endotoxicity. Transactions of the Royal Society of Tropical Medicine and Hygiene 76: 4-7

9. Davis T M, Suputtamongkol Y, Spencer J L, Ford S, Chienkul N, Schulenburg W E, White N J 1992 Measures of capillary permeability in acute falciparum malaria: relation to severity of infection and treatment. Clinical Infectious Diseases 15: 256-266

10. Freeman M J, Kirby B M, Panciera D L, Henik R A, Rosin E, Sullivan L J 1994 Hypotensive shock syndrome associated with acute Babesia canis infection in a dog. Journal of the American Veterinary Medical Association 204: 94-96

11. Gilles H M J 1951 Pathological processes in Babesia canis. BSc thesis, University of Oxford

12. Hardie E M 1995 Life-threatening bacterial infection. Compendium on Continuing Education for the Practising Veterinarian 17: 763-778

13. Hauptman J G, Walshaw R, Olivier N B 1997 Evaluation of the sensitivity and specificity of diagnostic criteria for sepsis in dogs. Veterinary Surgery 26: 393-397

14. Jacobson L S, Clark I A 1994 The pathophysiology of canine babesiosis: new approaches to an old puzzle. Journal of the South African Veterinary Association 65: 134-145

15. Jacobson L S, Swan G E 1995 Supportive treatment of canine babesiosis. Journal of the South African Veterinary Association 66: 95-105

16. King L G 1994 Postoperative complications and prognostic indicators in dogs and cats with septic peritonitis: 23 cases (1989-1992). Journal of the American Veterinary Medical Association 204: 407-414

17. Littman M P, Drobatz KJ 1995 Hypertensive and hypotensive disorders. In Ettinger S J, Feldman E C (eds) Textbook of veterinary internal medicine. W B Saunders, Philadelphia: 93-100 
18. Maegraith B, Gilles H M, Devakul K 1957 Pathological processes in Babesia canis infections. Zeitschrift für Tropenmedizin und Parasitologie 8: 485-514

19. Malherbe W D, Parkin B S 1951 Atypical symptomatology in Babesia canis infection. Journal of the South African Veterinary Association 22: 25-61

20. Mandell D C, King L G 1998 Fluid therapy in shock. Veterinary Clinics of North America: Small Animal Practice 28: 623-644

21. Michell A R 1985 What is shock? Journal of Small Animal Practice 26: 719-738

22. Natanson C, Eichenholz P W, Danner R L, Eichacker P Q, Hoffman W D, Kuo G C, Banks S M, MacVittie T J, Parrillo J E 1989 Endotoxin and tumor necrosis factor challenges in dogs simulate the cardiovascular profile of human septic shock. Journal of Experimental Medicine 169: 823-832

23. Parratt J R 1997 Nitric oxide - a key mediator in sepsis and endotoxaemia. Journal of Physiology and Pharmacology 48: 493-506

24. Porter J M, Ivatury R R 1998 In search of the optimal end points of resuscitation in trauma patients - a review. Journal of Trauma Injury Infection and Critical Care 44: 908-914

25. Reyers F, Leisewitz A L, Lobetti R G, Milner R J, Jacobson L S, Van Zyl M 1998 Canine babesiosis in South Africa - more than one disease. Does this serve as a model for falciparum malaria? Annals of Tropical Medicine and Parasitology 92: 503-511

26. Schetters T, Kleuskens J, Scholtes N, Gorenflot A 1998 Parasite localization and dissemination in the babesia-infected host. Annals of Tropical Medicine and Parasitology 92: 513-519

27. Schetters T P M, Moubri K, Precigout E, Kleuskens J, Scholtes N C, Gorenflot A 1997 Different Babesia canis isolates, different diseases. Parasitology 115: 485-493

28. Sitprija V, Napathorn S, Laorpatanaskul S, Suithichaiyakul T, Moollaor P, Suwangool P, Sridama V, Thamaree S, Tankeyoon M 1996 Renal and systemic hemodynamics in falciparum malaria. American Journal of Nephrology 16: 513-519

29. Statman R, Cheng W, Cunningham J N, Henderson J L, Damiani P, Siconolfi A, Rogers D, Horovitz J H 1994 Nitric oxide inhibition in the treatment of the sepsis syndrome is detrimental to tissue oxygenation. Journal of Surgical Research 57: 93-98

30. Supanaranond W, Davis T M E, Pukrittayakamee S, Nagachinta B, White N J 1993 Abnormal circulatory control in falciparum malaria: the effects of antimalarial drugs. European Journal of Clinical Pharmacology 44: 325-329

31. Taboada J, Merchant S R 1991 Babesiosis of companion animals and man. Veterinary
Clinics of North America: Small Animal Practice 21: 103-123

32. Tobias A H 1993 Noninvasive oscillometric blood pressure determination in the dog. MMedVet thesis, University of Pretoria

33. Tracey K J, Lowry S F, Fahey T J, Albert J D, Fong Y, Hesse D, Beutler B, Manogue K R, Calvano S, Wei H 1987 Cachectin/tumor necrosis factor induces lethal shock and stress hormone responses in the dog. Surgery, Gynecology and Obstetrics 164: 415-422

34. Vincent I C, Michell A R, Leahy R A 1993 Non-invasive measurement of arterial blood pressure in dogs: a potential indicator for the identification of stress. Research in Veterinary Science 54: 195-201

35. Warrell D A, Molyneux M E, Beales P F 1990 Severe and complicated malaria. Transactions of the Royal Society of Tropical Medicine and Hygiene 2: 1-65

36. White N J 1996 Malaria. In Cook G C (ed.) Manson's tropical diseases (8th edn). W B Saunders, London: 1087-1164

37. Wright I G 1977 Kinin, kininogen and kininase levels during acute Babesia bovis $(=B$. argentina $)$ infection of cattle. British Journal of Pharmacology 61: 567-572

38. Wright I G, Kerr J D 1977 Hypotension in acute Babesia bovis ( $=$ B. argentina) infections of splenectomized calves. Journal of Comparative Pathology 87: 531-537 